\title{
ON A GENERALIZATION OF THE MCCOY CONDITION
}

\author{
Young Cheol Jeon, Hong Kee Kim, Nam Kyun Kim, Tai Keun Kwak, \\ YANG LEe, AND Dong Eun YeO
}

\begin{abstract}
We in this note consider a new concept, so called $\pi$-McCoy, which unifies McCoy rings and IFP rings. The classes of McCoy rings and IFP rings do not contain full matrix rings and upper (lower) triangular matrix rings, but the class of $\pi$-McCoy rings contain upper (lower) triangular matrix rings and many kinds of full matrix rings. We first study the basic structure of $\pi$-McCoy rings, observing the relations among $\pi$-McCoy rings, Abelian rings, 2-primal rings, directly finite rings, and $(\pi$-)regular rings. It is proved that the $n$ by $n$ full matrix rings $(n \geq 2)$ over reduced rings are not $\pi$-McCoy, finding $\pi$-McCoy matrix rings over non-reduced rings. It is shown that the $\pi$-McCoyness is preserved by polynomial rings (when they are of bounded index of nilpotency) and classical quotient rings. Several kinds of extensions of $\pi$-McCoy rings are also examined.
\end{abstract}

\section{Basic properties of $\pi$-McCoy rings}

Throughout this note every ring is associative with identity unless otherwise stated. We use $R[x]$ to denote the polynomial ring with an indeterminate $x$ over $R$. Denote the $n$ by $n$ full matrix ring over $R$ by $\operatorname{Mat}_{n}(R)$ and the $n$ by $n$ upper (resp. lower) triangular matrix ring over $R$ by $U_{n}(R)$ (resp. $L_{n}(R)$ ). Note $\operatorname{Mat}_{n}(R)[x] \cong \operatorname{Mat}_{n}(R[x])$ and we will use this freely. Use $E_{i j}$ for the matrix with $(i, j)$-entry 1 and elsewhere $0 . \quad \mathbb{Z}_{n}$ denotes the ring of integers modulo $n$. The set of all nilpotent elements in $R$ is written by $N(R)$, and $N_{*}(R)$ denotes the prime radical of $R$.

McCoy [10] obtained the following in 1957. Given a commutative ring $R$

$$
f(x) g(x)=0 \text { implies } f(x) c=0 \text { for some nonzero } c \in R,
$$

Received March 10, 2009.

2000 Mathematics Subject Classification. 16N40, $16 \mathrm{U} 80$.

Key words and phrases. $\pi$-McCoy ring, McCoy ring, polynomial ring, matrix ring, classical quotient ring.

The first author was supported by (the) Korea Science Academy of KAIST Grant funded by the Korean Government(Ministry of Educaton, Science and Technology). The third author was supported by Hanbat National University in 2008. The fifth author was supported by the Korea Research Foundation Grant funded by the Korean Government (KRF-2008-521C00003). 
where $f(x)$ and $g(x)$ are nonzero polynomials in $R[x]$. Based on this result, Nielsen [11] called a ring $R$ (possibly non-commutative) right McCoy when the equation $f(x) g(x)=0$ implies $f(x) c=0$ for some nonzero $c \in R$, where $f(x), g(x)$ are nonzero polynomials in $R[x]$. Left McCoy rings are defined similarly. If a ring is both left and right McCoy, then the ring is called a McCoy ring.

Due to Cohn [5], a ring $R$ is called reversible if $a b=0$ implies $b a=0$ for $a, b \in R$. Anderson and Camillo [1], observing the rings whose zero products commute, used the term $Z C_{2}$ for what is called reversible. Reversible rings are McCoy by Nielsen [11, Theorem 2]. A ring $R$ is called reduced if $N(R)=0$. Reduced rings are reversible via a simple computation, and commutative rings are clearly reversible; hence they are McCoy. We use these facts freely.

We consider the following condition that is a generalization of the condition $(\dagger)$ (we see below). Given a ring $R$ consider a condition

(*) $f(x) g(x) \in N(R[x])$ implies $f(x) c \in N(R[x])$ for some nonzero $c \in R$, where $f(x)$ and $g(x)$ are nonzero polynomials in $R[x]$.

Proposition 1.1. (1) The condition $(*)$ is left-right symmetric.

(2) McCoy rings satisfy the condition $(*)$.

Proof. (1) Let $R$ be a ring satisfying the condition (*). Say $f(x) g(x) \in N(R[x])$ for nonzero polynomials $f(x), g(x)$ in $R[x]$. Then $g(x) f(x) \in N(R[x])$. Since $R$ satisfies the condition $(*), g(x) c \in N(R[x])$ for some nonzero $c \in R$ and so $c g(x) \in N(R[x])$. The converse can be proved similarly.

(2) Let $R$ be a McCoy ring and $f(x) g(x) \in N(R[x])$ for nonzero polynomials $f(x), g(x)$ in $R[x]$. Say that $(f(x) g(x))^{n}=0$ and $(f(x) g(x))^{n-1} \neq 0$ for some $n \geq 1$. Then there exists $m \geq 1$ such that $(g(x) f(x))^{m}=0$ and $(g(x) f(x))^{m-1} \neq 0$.

Case 1. $f(x) g(x)=0$ and $g(x) f(x)=0$

Since $R$ is right McCoy, there exist $a, b \in R \backslash 0$ such that $f(x) a=0$ and $g(x) b=0$.

Case 2. $f(x) g(x)=0$ and $g(x) f(x) \neq 0$

Since $R$ is McCoy and $f(x) g(x)=0$, there exist $a, b \in R \backslash 0$ such that $f(x) a=$ 0 and $b g(x)=0$.

Case 3. $f(x) g(x) \neq 0$ and $g(x) f(x)=0$

Since $R$ is McCoy and $g(x) f(x)=0$, there exist $a, b \in R \backslash 0$ such that $a f(x)=$ 0 and $g(x) b=0$.

Case 4. $f(x) g(x) \neq 0$ (then $n \geq 2)$ and $g(x) f(x) \neq 0$ (then $m \geq 2)$

Suppose that $f(x) g(x) \neq 0$ and $g(x) f(x) \neq 0$, and assume on the contrary that $f(x) \alpha \neq 0$ and $g(x) \alpha \neq 0$ for any nonzero $\alpha \in R$.

When $n=2$ we get $(g(x) f(x))^{n-1} \neq 0$, and so we put $n \geq 3$. If $(g(x) f(x))^{n-1}$ $=0$, then $g(x)\left((f(x) g(x))^{n-2} f(x)\right)=0$ implies $g(x) c=0$ for some $c \in R \backslash 0$ 
since $R$ is right McCoy and $(f(x) g(x))^{n-2} f(x) \neq 0$, a contradiction, entailing $(g(x) f(x))^{n-1} \neq 0$. So $g(x)(f(x) g(x))^{n-1}$ is either zero or nonzero, considering $(f(x) g(x))^{n}=0$.

If $g(x)(f(x) g(x))^{n-1} \neq 0$, then $f(x) d=0$ for some $d \in R \backslash 0$ since $R$ is right McCoy and $0=(f(x) g(x))^{n}=f(x)\left(g(x)(f(x) g(x))^{n-1}\right)$, a contradiction. So $g(x)(f(x) g(x))^{n-1}=0$.

If $g(x)(f(x) g(x))^{n-1}=0$, then $g(x) e=0$ for some $e \in R \backslash 0$ since $R$ is right McCoy and $(f(x) g(x))^{n-1} \neq 0$, a contradiction. So $g(x)(f(x) g(x))^{n-1} \neq 0$.

In any situation of Case 4 , we meet a contradiction. Therefore $f(x) \alpha=0$ for some $\alpha \in R \backslash 0$ or $g(x) \beta=0$ for some $\beta \in R \backslash 0$.

By Cases 1, 2, 3 and $4, f(x) \alpha \in N(R[x])$ or $\beta g(x) \in N(R[x])$ for some $\alpha, \beta \in R \backslash 0$. Thus $R$ satisfis the condition (*) by (1).

Based on Proposition 1.1, a ring will be called $\pi-M c C o y$ if it satisfies the condition $(*)$. McCoy rings are $\pi$-McCoy by Proposition 1.1(2); but the converse need not be true by the arguments, to follow. Note that the McCoyness is not left-right symmetric by $[11,4$. Final remarks], comparing with the symmetry of the $\pi$-McCoyness.

In the following we see basic examples for $\pi$-McCoy rings.

Lemma 1.2. (1) Let $R$ be a ring. If there exists a nonzero ideal $I$ of $R$ such that $I[x] \subseteq N(R[x])$, then $R$ is $\pi-M c C o y$.

(2) Every non-semiprime ring is $\pi-M c C o y$.

(3) Let $R$ be a ring with a nonzero nilpotent ideal. Then $\operatorname{Mat}_{n}(R)(n \geq 1)$ is $\pi$-McCoy.

(4) Let $R$ be any ring. $U_{n}(R)$ and $L_{n}(R)$ are $\pi-M c C o y$ when $n \geq 2$.

(5) Let $R, S$ be rings. For a bimodule ${ }_{R} M_{S}$ (resp. $\left.{ }_{S} M_{R}\right)\left(\begin{array}{cc}R & M \\ 0 & S\end{array}\right)$ (resp. $\left(\begin{array}{cc}R & 0 \\ M & S\end{array}\right)$ ) is $\pi-M c C o y$.

(6) Let $R$ be a ring and $n$ be any positive integer. Then $R[x] /\left(x^{n}\right)$ is a $\pi$-McCoy ring, where $\left(x^{n}\right)$ is the ideal generated by $x^{n}$.

(7) Let $R$ be a ring with a nonzero central element $a \in N(R)$. Then $\operatorname{Mat}_{n}(R)$ is $\pi$-McCoy.

Proof. (1) Let $0 \neq f \in R[x]$. If $f \in I[x]$, then $f r \in N(R[x])$ for all $r \in R$. If $f \notin I[x]$, then $f s \in I[x] \subseteq N(R[x])$ for all nonzero $s \in I$. Thus $R$ is $\pi$-McCoy.

(2) Let $R$ be a ring with $N_{*}(R) \neq 0$. Since $0 \neq N_{*}(R)[x]=N_{*}(R[x]) \subseteq$ $N(R[x]), R$ is $\pi$-McCoy by (1).

The rings mentioned in (3), (4), (5) and (6) are non-semiprime and so they are $\pi$-McCoy by (2).

(7) Let $M=\operatorname{Mat}_{n}(R)$ and $m=\left(m_{i j}\right) \in M$ with $m_{i i}=a$ for all $i$ and elsewhere zero. Then $M m M[x]$ is a nonzero nilpotent ideal of $M[x]$ and so $M$ is $\pi$-McCoy by (2).

There exist many semiprime rings which are not $\pi$-McCoy by Theorem 1.4 below. $\operatorname{Mat}_{n}\left(\mathbb{Z}_{m^{2}}\right)$ is $\pi$-McCoy by Lemma $1.2(7)$ for every positive integer 
$m \geq 2$. However there are non- $\pi$-McCoy matrix rings (e.g., $\operatorname{Mat}_{n}\left(\mathbb{Z}_{p}\right)$ with $p$ prime) by Theorem 1.4, to follow.

$U_{n}(R)$ and $L_{n}(R)(n \geq 2)$ are $\pi$-McCoy by Lemma 1.2(4). However the following shows that $U_{n}(R)$ and $L_{n}(R)$ are both neither left nor right McCoy when $n \geq 2$.

Example 1.3. Let $R$ be any ring and set $U=U_{n}(R)$. For the case of $n=2 k$ $(k=1,2, \ldots)$, let

$$
\begin{aligned}
& f(x)=\left(E_{11}+\cdots+E_{(2 k-1)(2 k-1)}\right)+\left(-E_{12}-\cdots-E_{(2 k-1)(2 k)}\right) x, \\
& g(x)=\left(E_{22}+\cdots+E_{(2 k)(2 k)}\right)+\left(E_{12}+\cdots+E_{(2 k-1)(2 k)}\right) x
\end{aligned}
$$

be in $U[x]$. For the case of $n=2 k+1(k=1,2, \ldots)$, let

$$
\begin{aligned}
& f(x)=\left(E_{11}+E_{33}+\cdots+E_{(2 k+1)(2 k+1)}\right)+\left(-E_{12}-\cdots-E_{(2 k+1)(2 k)}\right) x, \\
& g(x)=\left(E_{22}+\cdots+E_{(2 k)(2 k)}\right)+\left(E_{12}+\cdots+E_{(2 k+1)(2 k)}\right) x
\end{aligned}
$$

be in $U[x]$. Then $f(x) g(x)=0$. Assuming $f(x) M=0$ for $M \in U_{n}(R)$, we get $M=0$ by the coefficients of $f(x)$; hence $U_{n}(R)$ is not right McCoy. Taking similar polynomials, we can also conclude that $U_{n}(R)$ is not left McCoy. Similarly $L_{n}(R)$ is neither left nor right McCoy.

From Lemma 1.2(4) one may conjecture that the $n$ by $n$ full matrix ring over any ring is $\pi$-McCoy for $n \geq 2$. In the following we see that matrix rings need not be $\pi$-McCoy.

Theorem 1.4. Let $R$ be a reduced ring. Then the $n$ by $n$ full matrix ring over $R$ is not $\pi-M c$ Coy when $n \geq 2$.

Proof. Let $S=\operatorname{Mat}_{n}(R)$ with $n \geq 2$ and we will proceed by induction on $n$ to show the non- $\pi$-McCoyness of $S$. Considering polynomials

$$
f=\left(\begin{array}{cccc}
1 & x & \cdots & x^{n-1} \\
x^{n} & x^{n+1} & \cdots & x^{2 n-1} \\
\vdots & \vdots & \cdots & \vdots \\
x^{n(n-1)} & x^{n(n-1)+1} & \cdots & x^{n^{2}-1}
\end{array}\right) \text { and } g=\left(\begin{array}{cccc}
x & x & \cdots & x \\
-1 & -1 & \cdots & -1 \\
0 & 0 & \cdots & 0 \\
\vdots & \vdots & \cdots & \vdots \\
0 & 0 & \cdots & 0
\end{array}\right)
$$

in $S[x]$, we have $f g=0$. Let $C=\left(c_{i j}\right) \in S$ such that $f C \in N(S[x])$, say $(f C)^{k}=0$. Note

$$
f C=\left(\begin{array}{cccc}
h_{11} & h_{12} & \cdots & h_{1 n} \\
h_{11} x^{n} & h_{12} x^{n} & \cdots & h_{1 n} x^{n} \\
\vdots & \vdots & \cdots & \vdots \\
h_{11} x^{n(n-1)} & h_{12} x^{n(n-1)} & \cdots & h_{1 n} x^{n(n-1)}
\end{array}\right)
$$

where $h_{1 j}=\sum_{i=1}^{n} c_{i j} x^{i-1} \in R[x]$ for $j \in\{1,2, \ldots, n\}$. The $(1,1)$-entry of $(f C)^{k}$ is

$$
h_{11}^{k}+h_{11}^{k-2} h_{12} h_{11} x^{n}+\cdots+h_{11}^{k-2} h_{1 n} h_{11} x^{(n-1) n}+\cdots=c_{11}^{k}+x h=0
$$


for some $h \in R[x]$ and so $c_{11}=0$ by the reducedness of $R$, entailing $h_{11}=$ $c_{21} x+\cdots+c_{n 1} x^{n-1}$. Note $v k<v(k-1)+n$ for each $v \in\{1,2, \ldots, n-1\}$, and so the degree of the first term in any polynomial in

$$
\left\{h_{11}^{k-2} h_{12} h_{11} x^{n}, \ldots, h_{11}^{k-2} h_{1 n} h_{11} x^{(n-1) n}, \ldots\right\}
$$

is larger than the degree of $h_{11}^{k}$. Assuming that $c_{11}=\cdots=c_{s 1}=0$ for $s \in$ $\{1,2, \ldots, n\}$, we have that the $(1,1)$-entry of $(f C)^{k}$ is $c_{(s+1) 1}^{k} x^{s k}+x^{s k+1} p=0$ for some $p \in R[x]$ and so $c_{(s+1) 1}=0$ by the reducedness of $R$. Inductively we finally obtain $c_{11}=\cdots=c_{n 1}=0$, entailing

$$
f C=\left(\begin{array}{cccc}
0 & h_{12} & \cdots & h_{1 n} \\
0 & h_{12} x^{n} & \cdots & h_{1 n} x^{n} \\
\vdots & \vdots & \ldots & \vdots \\
0 & h_{12} x^{n(n-1)} & \cdots & h_{1 n} x^{n(n-1)}
\end{array}\right)
$$

Next assume that $c_{i j}=0$ for all $i \in\{1,2, \ldots, n\}$ and $j \in\{1, \ldots, s\}$, based on the matrix $(*)$. Then we will show that $c_{i(s+1)}=0$ for all $i \in\{1,2, \ldots, n\}$. Note that the $(1, s+1)$-entry of $(f C)^{k}$ is

$$
\begin{aligned}
& \left(h_{1(s+1)}^{k} x^{s n}+h_{1(s+1)}^{k-2} h_{1(s+2)} h_{1(s+1)} x^{(s+1) n}+\cdots\right. \\
& \left.+h_{1(s+1)}^{k-2} h_{1 n} h_{1(s+1)} x^{(n-1) n}\right) x^{(k-2) s n}+\cdots \\
= & c_{1(s+1)}^{k} x^{(k-1) s n}+x^{(k-1) s n+1} w=0
\end{aligned}
$$

for some $w \in R[x]$ and so $c_{1(s+1)}=0$ by the reducedness of $R$, entailing $h_{1(s+1)}=c_{2(s+1)} x+\cdots+c_{n(s+1)} x^{n-1}$. By the same method as the case of the $(1,1)$-entry, we obtain $c_{1(s+1)}=\cdots=c_{n(s+1)}=0$. Now the induction gives that all $c_{i j}$ 's are zero, concluding that $S$ is not $\pi$-McCoy.

By Lemma 1.2(3), if $R$ is non-semiprime, then $\operatorname{Mat}_{n}(R)(n \geq 2)$ is $\pi$-McCoy. Note that reduced rings are semiprime. From Theorem 1.4 one may suspect that the $n$ by $n$ matrix ring over a semiprime ring is not $\pi$-McCoy for $n \geq 2$. However the following example erases the possibility.

Example 1.5. Let $S$ be a reduced ring, $n$ be a positive integer and $R_{n}$ be the $2^{n}$ by $2^{n}$ upper triangular matrix ring over $S$. Define a map $\sigma: R_{n} \rightarrow R_{n+1}$ by $A \mapsto\left(\begin{array}{cc}A & 0 \\ 0 & A\end{array}\right)$, then $R_{n}$ can be considered as a subring of $R_{n+1}$ via $\sigma$ (i.e., $A=\sigma(A)$ for $\left.A \in R_{n}\right)$. Notice that $D=\left\{R_{n}, \sigma_{n m}\right\}$, with $\sigma_{n m}=\sigma^{m-n}$ whenever $n \leq m$, is a direct system over $I=\{1,2, \ldots\}$. Set $R=\lim R_{n}$ be the direct limit of $D$. Then $R$ is a semiprime ring by the method in $\overrightarrow{[8}$, Example 1.2]. Consider the following two-sided ideal

$$
I=\{A \in R \mid \text { the diagonal entries of } A \text { are zero }\} .
$$

Now let $J=\operatorname{Mat}_{n}(I)$ and $f(x)=A_{0}+A_{1} x+\cdots+A_{m} x^{m} \in J[x]$. Then there exists $k \geq 1$ such that $A_{i} \in R_{k}$ for all $i=0,1, \ldots, m$; hence $f(x)$ is nilpotent, entailing that $J[x]$ is nil. By Lemma 1.2(1), $\operatorname{Mat}_{n}(R)(n \geq 2)$ is $\pi$-McCoy. 
Note. Let $R$ be a right McCoy ring and suppose that $0 \neq f(x)=\sum_{i=0}^{m} a_{i} x^{i}$, $0 \neq g(x) \in R[x]$ satisfy $f(x) g(x)=0$. Then since $R$ is right McCoy, there is $0 \neq c \in R$ with $f(x) c=0$; hence $\left(\sum_{i=0}^{m} R a_{i}\right) c=0$ and so $\sum_{i=0}^{m} R a_{i}$ contains no right regular element (of course $\sum_{i=0}^{m} R a_{i} \varsubsetneqq R$ ). Thus if there exist $0 \neq$ $f(x)=\sum_{i=0}^{m} a_{i} x^{i}, 0 \neq g(x) \in R[x]$ with $f(x) g(x)=0$ and $\sum_{i=0}^{m} R a_{i}$ contains a right regular element, then $R$ cannot be right McCoy.

In the following we show by Note that any $n$ by $n(n \geq 2)$ full matrix ring is neither left nor right McCoy over any ring.

Proposition 1.6. Let $R$ be any ring. $\operatorname{Mat}_{n}(R)(n \geq 2)$ is neither left nor right McCoy.

Proof. Let $R$ be any ring and $M=\operatorname{Mat}_{n}(R)$ for any $n \geq 2$. For the case of $n=2 k(k=1,2, \ldots)$, let

$$
\begin{aligned}
& f(x)=\left(E_{12}+\cdots+E_{(2 k-1)(2 k)}\right)+\left(E_{11}+\cdots+E_{(2 k-1)(2 k-1)}\right) x, \\
& g(x)=\left(E_{12}+\cdots+E_{(2 k-1)(2 k)}\right)+\left(-E_{22}-\cdots-E_{(2 k)(2 k)}\right) x
\end{aligned}
$$

in $M[x]$. For the case of $n=2 k+1(k=1,2, \ldots)$, let

$$
\begin{aligned}
& f(x)=\left(E_{12}+\cdots+E_{(2 k+1)(2 k)}\right)+\left(E_{11}+E_{33}+\cdots+E_{(2 k+1)(2 k+1)}\right) x, \\
& g(x)=\left(E_{12}+\cdots+E_{(2 k+1)(2 k)}\right)+\left(-E_{22}-\cdots-E_{(2 k)(2 k)}\right) x
\end{aligned}
$$

in $M[x]$. Then we get $f(x) g(x)=0$, but $\sum_{i=0}^{1} M a_{i}$ contains the identity; hence $M$ is not right McCoy by Note. Similarly we also conclude that $M$ is not left McCoy.

Due to Bell [2], a ring $R$ is called IFP if $a b=0$ implies $a R b=0$. A ring $R$ is called 2-primal if $N_{*}(R)=N(R)$, according to Birkenmeier et al. [3]. Note that a ring $R$ is 2-primal if and only if $R / N_{*}(R)$ is reduced. It can be easily checked that reversible rings are IFP and IFP rings are 2-primal.

Proposition 1.7. 2-primal rings are $\pi-M c C o y$.

Proof. Let $R$ be a 2-primal ring. If $N_{*}(R)=0$, then $R$ is reduced and so $R$ is $\left(\pi\right.$-)McCoy. If $N_{*}(R) \neq 0$, then $R$ is also $\pi$-McCoy by Lemma $1.2(2)$.

Using the construction in Section 3 in [11], there can be an IFP ring that is not one-sided McCoy. A ring is called Abelian if every idempotent is central. Note that IFP rings are Abelian. The ring in [4, Theorem 7.1] is McCoy but not Abelain. Thus McCoy rings also need not be IFP. By Proposition 1.7, the concept of $\pi$-McCoyness unifies IFP rings and McCoy rings.

The converse of Proposition 1.7 need not hold. The $\pi$-McCoy ring $R$ in Example 1.5 is not 2-primal by [8, Example 1.2]. It is natural to ask whether 2-primal rings are one-sided McCoy. However the answer is negative as can be seen by upper triangular matrix rings over reduced rings. Consider $U=U_{n}(R)$ 
$(n \geq 2)$ over a reduced ring $R$. Then $U$ is 2-primal since $N(U)=N_{*}(U)$. But $U$ is neither left nor right McCoy by Example 1.3.

A ring $R$ is called directly finite if $a b=1$ implies $b a=1$ for $a, b \in R$. 2primal rings and right McCoy rings are directly finite by [3, Proposition 2.10] and [4, Theorem 5.2], respectively. So one may conjecture that $\pi$-McCoy rings are directly finite. However the following erases the possibility.

Example 1.8. Let $F$ be a field and $\mathbb{V}$ be an infinite dimensional vector space over $F$ with a basis $\left\{v_{1}, v_{2}, \ldots\right\}$. Consider the endomorphism ring $R=\operatorname{End}_{F}(\mathbb{V})$ and define $f, g \in R$ such that $f v_{1}=0, f v_{j}=v_{j-1}$ for $j=2,3, \ldots$ and $g v_{i}=v_{i+1}$ for $i=1,2, \ldots$. Then $f g=1$ but $g f \neq 1$. Now consider $U_{n}(R)$ for $n \geq 2$. Then $U_{n}(R)$ is $\pi$-McCoy by Lemma 1.2(4). Take $a=\left(a_{i j}\right)$ and $b=\left(b_{i j}\right)$ in $U_{n}(R)$ such that $a_{i i}=f$ for all $i$, elsewhere zero, and $b_{i i}=g$ for all $i$, elsewhere zero. Then $a b=1$ but $b a \neq 1$; hence $U_{n}(R)$ is not directly finite.

Abelian rings are clearly directly finite and so one may ask whether Abelian rings are $\pi$-McCoy. But the following gives a negative answer.

Example 1.9. There exists an Abelian ring that is not $\pi$-McCoy.

Let $A$ be the 3 by 3 full matrix ring over the power series ring $F[[t]]$ over a field $F$. Let

$$
\begin{gathered}
B=\left\{M=\left(m_{i j}\right) \in A \mid m_{i j} \in t F[[t]] \text { for } 1 \leq i, j \leq 2\right. \text { and } \\
\left.m_{i j}=0 \text { for } i=3 \text { or } j=3\right\}
\end{gathered}
$$

and

$$
C=\left\{M=\left(m_{i j}\right) \in A \mid m_{i i} \in F \text { and } m_{i j}=0 \text { for } i \neq j\right\} .
$$

Let $R$ be the subring of $A$ generated by $B$ and $C$. Let $F=\mathbb{Z}_{2}$. Note that every element of $R$ is of the form

$$
\left(\begin{array}{ccc}
a+f_{1} & f_{2} & 0 \\
f_{3} & a+f_{4} & 0 \\
0 & 0 & a
\end{array}\right) \text { for some } a \in F \text { and } f_{i} \in t F[[t]](i=1,2,3,4) .
$$

Consider two polynomials over $R$

$$
f(x)=\left(\begin{array}{ccc}
t & t x & 0 \\
t x^{2} & t x^{3} & 0 \\
0 & 0 & 0
\end{array}\right) \text { and } g(x)=\left(\begin{array}{ccc}
t x & t x & 0 \\
-t & -t & 0 \\
0 & 0 & 0
\end{array}\right) \in R[x] .
$$

Then $f(x) g(x)=0$ but there cannot exist $r \in R$ such that $f(x) r \in N(R[x])$, concluding that $R$ is not $\pi-\mathrm{McCoy}$.

Next we will show that $R$ is Abelian. Let $e^{2}=e$ for $e=\left(\begin{array}{ccc}a+f_{1} & f_{2} & 0 \\ f_{3} & a+f_{4} & 0 \\ 0 & 0 & a\end{array}\right) \in R$. Then we get $a^{2}=a$ and

$$
\begin{aligned}
f_{1}^{2}+f_{2} f_{3} & =f_{1}, \\
f_{1} f_{2}+f_{2} f_{4} & =f_{2}, \\
f_{1} f_{3}+f_{3} f_{4} & =f_{3}, \\
f_{2} f_{3}+f_{4}^{2} & =f_{4} .
\end{aligned}
$$


$\left(f_{1}+f_{4}\right) f_{2}=f_{2}$ from $(2)$, and so $f_{2}=0$ since $f_{i}$ 's are in $t F[[t]]$. Then $f_{1}=0$ follows from (1), entailing $f_{3}=0=f_{4}$ from (3) and (4). Consequently we have that every idempotent of $R$ is of the form $e=\left(\begin{array}{lll}a & 0 & 0 \\ 0 & a & 0 \\ 0 & 0 & a\end{array}\right) \in R$. Thus $R$ is Abelian.

A ring $R$ is called (von Neumann) regular if for each $a \in R$ there exists $x \in R$ such that $a=a x a$. Note that a ring $R$ is Abelian regular if and only if $R$ is reduced regular [6, Theorem 3.2]. Consequently a regular ring $R$ is reduced if and only if $R$ is reversible if and only if $R$ is Abelian if and only if $R$ is 2 -primal. Recall that the class of $\pi$-McCoy rings contains both McCoy rings and 2-primal rings. So one may hope that regular $\pi$-McCoy rings are both McCoy and 2-primal. But in the following we see a negative case.

Example 1.10. Let $S$ be an Abelian regular ring, $n$ be a positive integer, and $R_{n}$ be the $2^{n}$ by $2^{n}$ full matrix ring over $S$. Define a map $\sigma: R_{n} \rightarrow R_{n+1}$ by $A \mapsto\left(\begin{array}{cc}A & 0 \\ 0 & A\end{array}\right)$, then $R_{n}$ can be considered as a subring of $R_{n+1}$ via $\sigma$ (i.e., $A=\sigma(A)$ for $\left.A \in R_{n}\right)$. Notice that $D=\left\{R_{n}, \sigma_{n m}\right\}$, with $\sigma_{n m}=\sigma^{m-n}$ whenever $n \leq m$, is a direct system over $I=\{1,2, \ldots\}$. Set $R=\lim R_{n}$ be the direct limit of $D$.

For $f(x), g(x) \in R[x]$ with $f(x) g(x)=0$, assume on the contrary that there exists $0 \neq c \in R$ with $f(x) c=0$. There exists $m \geq 1$ such that $f(x), g(x), c \in$ $R_{m}[x]$. However Proposition 1.6 implies that $R_{m}$ cannot be neither left nor right McCoy, a contradiction. Thus $R$ is not right McCoy. Similarly $R$ is not left McCoy. Next it is clear that $R$ is not 2-primal since $E_{12}+E_{21}$ is not nilpotent.

Now we claim that $R$ is $\pi$-McCoy. Assume $f(x) g(x) \in N(R[x])$ for $0 \neq$ $f(x), g(x) \in R[x]$. Then there exists $k \geq 1$ such that $f(x), g(x) \in R_{k}[x]$, and so we consider the matrix $E_{1\left(2^{k+1}\right)}$ in $R_{k+1}$. It then follows $\left(f(x) E_{1\left(2^{k+1}\right)}\right)^{2^{k+1}}=0$, obtaining that $R$ is $\pi$-McCoy.

Lastly in this section we observe subrings and homomorphic images of $\pi$ McCoy rings.

Proposition 1.11. (1) The class of $\pi-M c C o y$ rings is not closed under subrings.

(2) The class of $\pi$-McCoy rings is not closed under homomorphic images.

Proof. (1) Let $R$ be the ring, in Theorem 1.4, which is not $\pi$-McCoy. Next consider $U_{2}(R)$. Then $U_{2}(R)$ is $\pi$-McCoy by Lemma $1.2(4)$, but $R$ is a subring of $U_{2}(R)$.

(2) Let $R$ be the ring of quaternions with integer coefficients. Then $R$ is a domain, so $(\pi-)$ McCoy. However for any odd prime integer $q$, the ring $R / q R$ is isomorphic to $\operatorname{Mat}_{2}\left(\mathbb{Z}_{q}\right)$ by the argument in [7, Exercise $\left.2 \mathrm{~A}\right]$. Thus $R / q R$ is not $\pi$-McCoy by Theorem 1.4 when $q \in\{3,5,11\}$.

Let $S$ be a reduced ring and set $A=U_{n}(S), B=\operatorname{Mat}_{n}(S)$ for $n \geq 2$. Then $R=\left(\begin{array}{cc}A & B \\ 0 & B\end{array}\right)$ is $\pi$-McCoy by Lemma $1.2(4)$; but $\frac{R}{I} \cong \operatorname{Mat}_{n}(S)$ is not $\pi$-McCoy 
by Theorem 1.4 when $I=\left(\begin{array}{cc}A & B \\ 0 & 0\end{array}\right)$. This ring is another counterexample for Proposition 1.11(2).

\section{More examples of $\pi$-McCoy rings}

In this section we examine several kinds of extensions of $\pi$-McCoy rings. We first consider the case of classical quotient rings. A ring $R$ is called right Ore if given $a, b \in R$ with $b$ regular there exist $a_{1}, b_{1} \in R$ with $b_{1}$ regular such that $a b_{1}=b a_{1}$. It is well-known that $R$ is a right Ore ring if and only if the classical right quotient ring of $R$ exists.

Theorem 2.1. Let $R$ be a right Ore ring with its classical right quotient ring $Q$. If $R$ is $\pi-M c C o y$, then so is $Q$.

Proof. Denote the set of all regular elements in $R$ by $C(R)$. We use [9, Proposition 2.1.16] freely. Suppose $F(x) G(x) \in N(Q[x])$ for $F(x), G(x) \in Q[x] \backslash 0$. Say that $(F(x) G(x))^{k}=0$ and $(F(x) G(x))^{k-1} \neq 0$ for some $k \geq 1$. We can write $F(x)=\sum_{i=0}^{m} a_{i} u^{-1} x^{i}$ and $G(x)=\sum_{j=0}^{n} b_{j} v^{-1} x^{j}$ for some $a_{i}$ 's, $b_{j}$ 's in $R$ and $u, v \in C(R)$.

Case 1. $F(x) G(x)=0$ and $G(x) F(x)=0$

Consider $F(x) G(x)=0$. Since $R$ is right Ore, $u^{-1} b_{j}=b_{j}^{\prime} u_{1}^{-1}(j=1, \ldots, n)$ for some $b_{j}^{\prime} \in R$ and $u_{1} \in C(R)$. Letting $f(x)=\sum_{i=0}^{m} a_{i} x^{i}$ and $g_{1}(x)=$ $\sum_{j=0}^{n} b_{j}^{\prime} x^{j}$, we have $f(x) g_{1}(x)=0$. Since $R$ is $\pi$-McCoy, there exists $\alpha \in R \backslash 0$ with $f(x) \alpha \in N(R[x]) \subseteq N(Q[x])$ and hence

$$
F(x) u \alpha=f(x) u^{-1} u \alpha=f(x) \alpha \in N(Q[x])
$$

for some nonzero $u \alpha \in Q$.

For the case of $G(x) F(x)=0$, a similar computation finds nonzero $v \beta \in Q$ such that $G(x) v \beta \in N(Q[x])$.

Case 2. $F(x) G(x)=0$ and $G(x) F(x) \neq 0$

Note that $G(x) F(x) G(x)=0$ and $F(x) G(x) F(x)=0$. Letting $H(x)=$ $G(x) F(x)$, we have $H(x) G(x)=0$ and $F(x) H(x)=0$ with $H(x) \neq 0$. Say $H(x)=\sum_{s=0}^{\ell} c_{s} w^{-1} x^{s}$ with $c_{s}$ 's in $R$ and $w \in C(R)$.

Consider $H(x) G(x)=0$. Since $R$ is right Ore, $w^{-1} b_{j}=d_{j} w_{1}^{-1}$ for some $d_{j}$ 's in $R$ and $w_{1} \in C(R)$. Letting $h(x)=\sum_{s=0}^{\ell} c_{s} x^{s}$ and $g_{2}(x)=\sum_{j=0}^{n} d_{j} x^{j}$, we have $h(x) g_{2}(x)=0$. Since $R$ is $\pi$-McCoy, there exists $\gamma \in R \backslash 0$ with $\gamma g_{2}(x) \in$ $N(R[x]) \subseteq N(Q[x])$. Note $g_{2}(x) \gamma \in N(Q[x])$ and

$$
w^{-1} G(x) w_{1} \gamma=g_{2}(x) w_{1}^{-1} w_{1} \gamma=g_{2}(x) \gamma \in N(Q[x]) .
$$

It then follows that $G(x) w_{1} \gamma w^{-1} \in N(Q[x])$ for some nonzero $w_{1} \gamma w^{-1} \in Q$.

For the case of $F(x) H(x)=0$, the computation in Case 1 finds nonzero $q \in Q$ such that $F(x) q \in N(Q[x])$.

Case 3. $F(x) G(x) \neq 0$ and $G(x) F(x)=0$ 
Note that $F(x) G(x) F(x)=0$ and $G(x) F(x) G(x)=0$. Letting $I(x)=$ $F(x) G(x)$, we have $I(x) F(x)=0$ and $G(x) I(x)=0$ with $I(x) \neq 0$.

Then the computation in Case 2 gives $q_{1}, q_{2} \in Q \backslash 0$ such that $F(x) q_{1}$, $G(x) q_{2} \in N(Q[x])$.

Case 4. $F(x) G(x) \neq 0$ and $G(x) F(x) \neq 0$

Suppose $F(x) G(x) \neq 0$ and $G(x) F(x) \neq 0$. Then there exist $k \geq 2$ and $h \geq 2$ such that $(F(x) G(x))^{k}=0,(F(x) G(x))^{k-1} \neq 0,(G(x) F(x))^{h}=0$ and $(G(x) F(x))^{h-1} \neq 0$.

Assume on the contrary that $F(x) \alpha \notin N(Q[x])$ and $G(x) \alpha \notin N(Q[x])$ for any nonzero $\alpha \in Q$.

Since $R$ is right Ore, $u^{-1} b_{j}=b_{j}^{\prime} u_{1}^{-1}$ and $v^{-1} a_{i}=a_{i}^{\prime} v_{1}^{-1}$ for some $a_{i}^{\prime}, b_{j}^{\prime} \in R$ and $u_{1}, v_{1} \in C(R)$. Letting $f(x)=\sum_{i=0}^{m} a_{i} x^{i}, f_{1}(x)=\sum_{i=0}^{m} a_{i}^{\prime} x^{i}, g(x)=$ $\sum_{j=0}^{n} b_{j} x^{j}$ and $g_{1}(x)=\sum_{j=0}^{n} b_{j}^{\prime} x^{j}$, we obtain $F(x) G(x)=f(x) g_{1}(x)\left(v u_{1}\right)^{-1}$ and $G(x) F(x)=g(x) f_{1}(x)\left(u v_{1}\right)^{-1}$. Let $v_{2}=u v_{1}$ and $u_{2}=v u_{1}$. Also since $R$ is right Ore, $u_{2}^{-1}(F(x) G(x))^{k-1}=A(x) u_{3}^{-1}$ and $v_{2}^{-1}(G(x) F(x))^{h-1}=B(x) v_{3}^{-1}$ for some $A(x), B(x) \in R[x]$ and some $u_{3}, v_{3} \in C(R)$. Here $A(x)$ and $B(x)$ are both nonzero because $(F(x) G(x))^{k-1}$ and $(G(x) F(x))^{h-1}$ are both nonzero. Notice that

$$
f(x) g_{1}(x) A(x)=f(x) g_{1}(x) u_{2}^{-1}(F(x) G(x))^{k-1} u_{3}=(F(x) G(x))^{k} u_{3}=0
$$

and

$$
g(x) f_{1}(x) B(x)=g(x) f_{1}(x) v_{2}^{-1}(G(x) F(x))^{h-1} v_{3}=(G(x) F(x))^{h} v_{3}=0 .
$$

But $A(x)$ and $B(x)$ are both nonzero. So there are $\beta, \gamma \in R \backslash 0$ such that $f(x) g_{1}(x) \beta \in N(R[x])$ and $g(x) f_{1}(x) \gamma \in N(R[x])$, since $R$ is $\pi$-McCoy. Note that

and

$$
g_{1}(x) \beta=g_{1}(x) u_{2}^{-1} u_{2} \beta=u^{-1} g(x) v^{-1} u_{2} \beta=u^{-1} G(x) u_{2} \beta
$$

$$
f_{1}(x) \gamma=f_{1}(x) v_{2}^{-1} v_{2} \gamma=v^{-1} f(x) u^{-1} v_{2} \gamma=v^{-1} F(x) v_{2} \gamma
$$

Thus if $f_{1}(x) \gamma \in N(Q[x])$ and $g_{1}(x) \beta \in N(Q[x])$, then $F(x) v_{2} \gamma v^{-1} \in N(Q[x])$ and $G(x) u_{2} \beta u^{-1} \in N(Q[x])$ with $v_{2} \gamma v^{-1}, u_{2} \beta u^{-1} \in Q \backslash 0$. This is a contradiction to assumption, entailing $f_{1}(x) \gamma \notin N(Q[x])$ and $g_{1}(x) \beta \notin N(Q[x])$. Since $R$ is $\pi$-McCoy, $f(x) \delta \in N(R[x])$ and $g(x) \zeta \in N(R[x])$ for some $\delta, \zeta \in$ $R \backslash 0$. It then follows that $F(x) u \delta=f(x) u^{-1} u \delta=f(x) \delta \in N(R[x])$ and $G(x) v \zeta=g(x) v^{-1} v \zeta=g(x) \zeta \in N(R[x])$ with $u \delta, v \zeta \in Q \backslash 0$, a contradiction to the assumption. Therefore $F(x) \alpha \in N(Q[x])$ or $G(x) \beta \in N(Q[x])$ for some $\alpha, \beta \in Q \backslash 0$.

By Cases 1, 2, 3 and $4, F(x) \alpha \in N(Q[x])$ or $\beta G(x) \in N(Q[x])$ for some $\alpha, \beta \in Q \backslash 0$. Thus $Q$ is $\pi$-McCoy.

Next we observe the case of polynomial rings.

Theorem 2.2. Let $R$ be a ring and suppose that $(f c)^{h}=0$ for some $0 \neq c \in R$ and $h \leq k$ whenever $(f g)^{k}=0$ for nonzero $f, g$ in $R[x]$. Then $R[x]$ is $\pi$-McCoy. 
Proof. Let $S=R[x]$. Let $R$ be $\pi$-McCoy and suppose that $f(t) g(t) \in N(S[t])$ for nonzero polynomials $f(t)=f_{0}+f_{1} t+\cdots+f_{m} t^{m}$ and $g(t)=g_{0}+g_{1} t+\cdots+$ $g_{n} t^{n}$ in $S[t]$, where $S[t]$ is the polynomial ring with an indeterminate $t$ over $S$. Say $(f(t) g(t))^{\ell}=0$. Set $k=\operatorname{deg}\left(f_{0}\right)+\cdots+\operatorname{deg}\left(f_{m}\right)+\operatorname{deg}\left(g_{0}\right)+\cdots+\operatorname{deg}\left(g_{n}\right)$ and replace $t$ by $x^{h}$ with $h=k \ell+1$. Then the set of coefficients of the $f_{i}$ 's and $g_{j}$ 's coincides with the set of coefficients of $f\left(x^{h}\right)$ and $g\left(x^{h}\right)$; hence $f\left(x^{h}\right)$ and $g\left(x^{h}\right)$ are nonzero polynomials in $R[x]$. Moreover we have

$$
\left(f\left(x^{h}\right) g\left(x^{h}\right)\right)^{\ell}=\sum_{s=0}^{(m+n) \ell} \sum_{i_{1}+\cdots+i_{\ell}=s} p_{i_{1}} q_{i_{1}} \cdots p_{i_{\ell}} q_{i_{\ell}}\left(x^{h}\right)^{s},
$$

where $p_{i_{j}} \in\left\{f_{0}, \ldots, f_{m}\right\}$ and $q_{i_{j}} \in\left\{g_{0}, \ldots, g_{n}\right\}$ for all $i_{j}$ 's. But these

$$
p_{i_{1}} q_{i_{1}} \cdots p_{i_{\ell}} q_{i_{\ell}} \text { 's }
$$

are equal to those of $(f(t) g(t))^{\ell}$, and so $\left(f\left(x^{h}\right) g\left(x^{h}\right)\right)^{\ell}=0$. Now by hypothesis, $\left(f\left(x^{h}\right) c\right)^{v}=0$ for some $0 \neq c \in R$ and $v \leq \ell$. This implies that $(f(t) c)^{v}=0$ with $0 \neq c \in S$ since the coefficients of $\left(f\left(x^{h}\right) c\right)^{v}=0$ and $(f(t) c)^{v}=0$ are equal. Thus $R[x]$ is $\pi$-McCoy.

Proposition 2.3. A ring $R$ is $\pi-M c C o y$ if $R[x]$ is $\pi-M c C o y$.

Proof. Let $S=R[x]$ be $\pi$-McCoy and suppose that $f(x) g(x) \in N(R[x])$ for nonzero polynomials $f(x), g(x)$ in $R[x]$. This can be converted to that $f(t) g(t) \in N(S[t])$ for nonzero polynomials $f(t), g(t)$ in $S[t]$. Since $S$ is $\pi$ McCoy, $f(t) h(x) \in N(S[t])$ for some $0 \neq h(x) \in R[x]$, say $(f(t) h(x))^{k}=0$. Note that $f(t) \in R[t]$. Here letting $h(x)=\sum_{i=0}^{n} a_{i} x^{i} \in R[x]$ (we can set $a_{0} \neq 0$, dividing by $x$ if necessary), then we get $f(t) a_{0} \in N(R[t])$ from $0=(f(t) h(x))^{k}=\left(f(t) a_{0}\right)^{k}+h_{1} x+\cdots+h_{n k} x^{n k}$ with $h_{1}, \ldots, h_{n k} \in R[t]$. This implies that $f(x) a_{0} \in N(R[x])$ and $0 \neq a_{0} \in R$, showing that $R$ is $\pi$ McCoy.

The index of nilpotency of a nilpotent element $x$ in a ring $R$ is the least positive integer $n$ such that $x^{n}=0$. The index of nilpotency of a subset $I$ of $R$ is the supremum of the indices of nilpotency of all nilpotent elements in $I$. If such a supremum is finite, then $I$ is said to be of bounded index of nilpotency.

From Theorem 2.2 and Proposition 2.3 we can obtain the following.

Corollary 2.4. Let $R$ be a ring of bounded index of nilpotency. Then $R$ is $\pi-M c C o y$ if and only if $R[x]$ is $\pi-M c C o y$.

In the following we deal with a case similar to the classical quotient rings.

Proposition 2.5. Let $R$ be a ring and $\Delta$ be a multiplicatively closed subset of $R$ consisting of central regular elements. Then $R$ is $\pi-M c C o y$ if and only if $\Delta^{-1} R$ is $\pi$-McCoy. 
Proof. Suppose that $R$ is $\pi$-McCoy. Let $F(x)=\sum_{i=0}^{m} \alpha_{i} x^{i}$ and $G(x)=$ $\sum_{j=0}^{n} \beta_{j} x^{j}$ be nonzero polynomials in $\left(\Delta^{-1} R\right)[x]$ such that

$$
F(x) G(x) \in N\left(\left(\Delta^{-1} R\right)[x]\right),
$$

where $\alpha_{i}=a_{i} u^{-1}, \beta_{j}=b_{j} v^{-1}$ with $a_{i}, b_{j} \in R$ and $u, v \in \Delta$. Since $\Delta$ is contained in the center of $R$, we have $F(x) G(x)=u^{-1}\left(a_{0}+a_{1} x+\cdots+\right.$ $\left.a_{m} x^{m}\right) v^{-1}\left(b_{0}+b_{1} x+\cdots+b_{n} x^{n}\right)=(u v)^{-1}\left(a_{0}+a_{1} x+\cdots+a_{m} x^{m}\right)\left(b_{0}+\right.$ $\left.b_{1} x+\cdots+b_{n} x^{n}\right) \in N\left(\left(\Delta^{-1} R\right)[x]\right)$. Let $f(x)=a_{0}+a_{1} x+\cdots+a_{m} x^{m}$ and $g(x)=b_{0}+b_{1} x+\cdots+b_{n} x^{n}$. Then $f(x)$ and $g(x)$ are nonzero polynomials in $R[x]$ with $f(x) g(x) \in N(R[x])$. Since $R$ is right $\pi$-McCoy, there exists $0 \neq c \in R$ such that $f(x) c \in N(R[x])$. Hence $F(x) c=u^{-1} f(x) c \in N\left(\left(\Delta^{-1} R\right)[x]\right)$, concluding that $\Delta^{-1} R$ is $\pi$-McCoy.

Conversely assume that $\Delta^{-1} R$ is $\pi$-McCoy. Let $f(x)=\sum_{i=0}^{m} a_{i} x^{i}$ and $g(x)=$ $\sum_{j=0}^{n} b_{j} x^{j}$ be nonzero polynomials in $R[x]$ such that $f(x) g(x) \in N(R[x])$. Since $\Delta^{-1} R$ is $\pi$-McCoy, $f(x)\left(c w^{-1}\right) \in N\left(\left(\Delta^{-1} R\right)[x]\right)$ for some $0 \neq c w^{-1} \in \Delta^{-1} R$. Thus we get $f(x) c \in N(R[x])$ and $0 \neq c \in R$, showing that $R$ is $\pi$-McCoy.

Corollary 2.6. Let $R$ be a ring. Then $R[x]$ is $\pi$-McCoy if and only if so is $R\left[x ; x^{-1}\right]$.

Proof. Note that $\Delta=\left\{1, x, x^{2}, \ldots\right\}$ is a multiplicatively closed subset of $R[x]$ consisting of central regular elements. So Proposition 2.5 gives the proof since $R\left[x ; x^{-1}\right]=\Delta^{-1} R[x]$.

We consider several basic examples as follows.

Proposition 2.7. Let $\left\{R_{i} \mid i \in I\right\}$ be a class of rings.

(1) The direct product and direct sum of $R_{i}$ 's are $\pi$-McCoy when some $R_{i}$ is non-semiprime.

(2) Suppose that $N_{*}\left(R_{i}\right)=0$ for all $i \in I$. The direct product of $R_{i}$ 's is $\pi-M c C o y$ if $R_{i}$ is $\pi-M c C o y$ for all $i \in I$.

(3) Suppose that $N_{*}\left(R_{i}\right)=0$ for all $i \in I$. The direct sum of $R_{i}$ 's is $\pi-M c C o y$ if $R_{i}$ is $\pi-M c$ Coy for all $i \in I$.

(4) The direct sum of $R_{i}$ 's is $\pi-M c$ Coy if $|I|=\infty$.

Proof. (1) If some $R_{i}$ is non-semiprime, then the direct product and direct sum of $R_{i}$ 's are also non-semiprime; hence they are $\pi$-McCoy by Lemma 1.2(2).

(2) Let $R$ be the direct product of $R_{i}$ and suppose $f(x) g(x) \in N(R[x])$ with $0 \neq f(x)=\sum_{j=0}^{m}\left(a(j)_{i}\right) x^{j}, 0 \neq g(x)=\sum_{k=0}^{n}\left(b(k)_{i}\right) x^{k} \in R[x]$. Letting $f_{i}(x)=\sum_{j=0}^{m} a(j)_{i} x^{j}$ and $g_{i}(x)=\sum_{k=0}^{n} b(k)_{i} x^{k}$ we can write $f(x)=\left(f_{i}(x)\right)$ and $g(x)=\left(g_{i}(x)\right)$.

If $f_{h}(x)=0$ for some $h \in I$, then take $r=\left(r_{i}\right) \in R$ be such that $r_{h}=1$ and zero elsewhere, obtaining $f(x) r=0$ with $r \neq 0$. So assume that $f_{i}(x) \neq 0$ for all $i \in I$. Since $g(x) \neq 0$, there exists $k \in I$ such that $g_{k}(x) \neq 0$. Since $R_{k}$ is $\pi$-McCoy by hypothesis, there exists $0 \neq c \in R_{k}$ such that $f_{k}(x) c \in N\left(R_{k}[x]\right)$. 
Let $r=\left(r_{i}\right) \in R$ be such that $r_{k}=c$ and zero elsewhere. Then $f(x) r \in N(R[x])$ with $r \neq 0$, concluding that $R$ is $\pi$-McCoy.

(3) The proof is almost same as (2).

(4) Let $R$ be the direct sum and $0 \neq f(x)=\sum_{j=0}^{m}\left(a(j)_{i}\right) x^{j} \in R[x]$. Since $|I|=\infty$, there exists $k \in I$ such that $a(j)_{k}=0$, the coordinate of $\left(a(j)_{i}\right)$ in $R_{k}$, for all $j$. Take $r=\left(r_{i}\right) \in R$ be such that $r_{k}=1$ and zero elsewhere. Then $f(x) r=0$ with $r \neq 0$ and so $R$ is $\pi$-McCoy.

Next we see the direct limit case.

Proposition 2.8. The classes of $\pi-M c C o y$ rings and right (or left) McCoy rings are closed under direct limits.

Proof. Let $D=\left\{R_{i}, \alpha_{i j}\right\}$ be a direct system of $\pi$-McCoy rings $R_{i}$ for $i \in I$ and ring homomorphisms $\alpha_{i j}: R_{i} \rightarrow R_{j}$ for each $i \leq j$ satisfying $\alpha_{i j}(1)=1$, where $I$ is a directed partially ordered set. Set $R=\lim R_{i}$ be the direct limit of $D$ with $\iota_{i}: R_{i} \rightarrow R$ and $\iota_{j} \alpha_{i j}=\iota_{i}$. We will show that $R$ is a $\pi$-McCoy ring. Take $a, b \in R$. Then $a=\iota_{i}\left(a_{i}\right), b=\iota_{j}\left(b_{j}\right)$ for some $i, j \in I$ and there is $k \in I$ such that $i \leq k, j \leq k$. Define

$$
a+b=\iota_{k}\left(\alpha_{i k}\left(a_{i}\right)+\alpha_{j k}\left(b_{j}\right)\right) \text { and } a b=\iota_{k}\left(\alpha_{i k}\left(a_{i}\right) \alpha_{j k}\left(b_{j}\right)\right),
$$

where $\alpha_{i k}\left(a_{i}\right)$ and $\alpha_{j k}\left(b_{j}\right)$ are in $R_{k}$. Then $R$ forms a ring with $0=\iota_{i}(0)$ and $1=\iota_{i}(1)$.

Now let $f, g \in R[x]$ be nonzero polynomials such that $f g \in N(R[x])$. There is $k \in I$ such that $f, g \in R_{k}[x]$ via $\iota_{i}$ 's and $\alpha_{i j}$ 's; hence we get $f g \in N\left(R_{k}[x]\right)$. Since $R_{k}$ is $\pi$-McCoy, there exists $0 \neq c_{k} \in R_{k}$ such that $f c_{k} \in N\left(R_{k}[x]\right)$. Put $c=\iota_{k}\left(c_{k}\right)$. Then $f c \in N(R[x])$ with nonzero $c$, entailing $R$ being $\pi$-McCoy. The proof for right (or left) McCoy rings is similar.

\section{References}

[1] D. D. Anderson and V. Camillo, Semigroups and rings whose zero products commute, Comm. Algebra 27 (1999), no. 6, 2847-2852.

[2] H. E. Bell, Near-rings in which each element is a power of itself, Bull. Austral. Math. Soc. 2 (1970), 363-368.

[3] G. F. Birkenmeier, H. E. Heatherly, and E. K. Lee, Completely prime ideals and associated radicals, Ring theory (Granville, OH, 1992), 102-129, World Sci. Publ., River Edge, NJ, 1993.

[4] V. Camillo and P. P. Nielsen, McCoy rings and zero-divisors, J. Pure Appl. Algebra 212 (2008), no. 3, 599-615.

[5] P. M. Cohn, Reversible rings, Bull. London Math. Soc. 31 (1999), no. 6, 641-648.

[6] K. R. Goodearl, von Neumann Regular Rings, Monographs and Studies in Mathematics, 4. Pitman (Advanced Publishing Program), Boston, Mass.-London, 1979.

[7] K. R. Goodearl and R. B. Warfield, Jr., An Introduction to Noncommutative Noetherian Rings, Cambridge University Press, Cambridge, 1989.

[8] S. U. Hwang, Y. C, Jeon, and Y. Lee, Structure and topological conditions of NI rings, J. Algebra 302 (2006), no. 1, 186-199.

[9] J. C. McConnell and J. C. Robson, Noncommutative Noetherian Rings, With the cooperation of L. W. Small. Pure and Applied Mathematics (New York). A Wiley-Interscience Publication. John Wiley \& Sons, Ltd., Chichester, 1987. 
[10] N. H. McCoy, Annihilators in polynomial rings, Amer. Math. Monthly 64 (1957), 28-29.

[11] P. P. Nielsen, Semi-commutativity and the McCoy condition, J. Algebra 298 (2006), no. $1,134-141$.

Young CheOl JeON

Department of Mathematics

Korea Science ACAdemy

BUSAN 614-103, KOREA

E-mail address: jachun@chol.com

Hong KeE Kim

Department of Mathematics and RINS

Gyeongsang National University

Jinju 660-701, KoreA

E-mail address: hkkim@gsnu.ac.kr

NAM KYUN Kim

College of Liberal Arts

Hanbat National University

DAEJEON 305-719, KOREA

E-mail address: nkkim@hanbat.ac.kr

Tai Keun Kwak

Department of Mathematics

DAEJIN UnIVERSITY

Pocheon 487-711, Korea

E-mail address: tkkwak@daejin.ac.kr

YANG LEE

Department of Mathematics

Busan National University

BUSAN 609-735, KOREA

E-mail address: ylee@pusan.ac.kr

Dong Eun Yeo

Department of Mathematics

Busan National University

Busan 609-735, Korea

E-mail address: dongeunyeo@pusan.ac.kr 